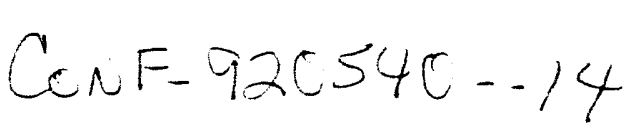

\section{A CAPACITANCE-BASED PROXIMITY SENSOR FOR WHOLE ARM OBST ACLE AVOIDANCE}

\author{
J. L. Novak and J. T. Feddema
}

Sandia National Laboratories

Albuquerque, NM 87185 USA
SAND- $-91-2119 C$

DE92 000820

\title{
Abstract
}

This paper addresses the issue of collision avoidance in unknown or partially modeled environments using a capacitive sensor. An eight channel capacitance-based sensor system which can detect obstacles up to $400 \mathrm{~mm}$ (16 inches) away has been developed. This sensor can detect both conductive and non-conductive obstacles of arbitrary color and shape. The sensor hardware is reliable and inexpensive, and it may be fabricated lising flexible printed circuit boards to provide whole-arm and joint protection for any robot or manipulator. Simple collision avoidance control algorithms have been implemented on a two-link robot arm. The sensor and control system enable the robot arm to avoid a conductive post and a concrete block.

\section{Introduction}

Much of the current robotics effort at the US Department of Energy is directed towird remote processing of hazardous waste. Some of this waste threatens the environment. requiring that active steps be taken to stabilize, detoxify, or repackage the material. An important tenet of suitable waste handling schemes is that the act of processing the wiste must create no additional hazards and do no damage to the containment structure. For example, in one application a 40 toot robot manipulator processing waste inside a closed vessel must not be allowed to puncture the tank wall or to bend vertical thermocouple trees which are present throughout the vessel.

One technique of preventing collisions, especially along the length of a robot link. involves the use of proximity sensors located such that the active regions encompass the entire rohor surface $[1,2,3]$. Additionally, since it is desirable to continue purposeful motion in the presence of obstacles, the sensor system must be able to deliver spatially-resslved proximity data which reflects the distance to the obstacle, as well as the location along the robut anil

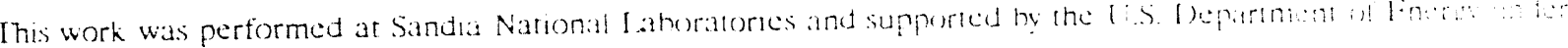
(ontract DFACO-76) 
corresponding robot surface normal. This vector information may then be used to modify trajectories to permit (if possible) continued progress toward the final destination.

Useful algorithms exist for perturbing trajectories based upon encountered obstacles. Lumelsky and Cheung [4] compute the plane tangent to an obstacle sensed by infrared photodetectors and then restrict the robot motion to this plane. Tilove [5] surveys the various local methods using artificial potentials to avoid collisions. These include the notion of a generalized potential field [6], in which the perturbing force depends on thi velocity as well as the position. All of these techniques are independent of the type of sensor used.

\section{Capacitive proximity sensors}

Capacitance-based proximity sensing for collision avoidance offers many advantages. The distribution of the electric field allows broad coverage of a robot without large numbers of sensors. Changes in the electric field due to obstacles are sensed instantaneously, unlike sonar-based systems which require listenirg for a return echo. Additionally, the capacitancr. measurement is .nsensitive to the color, texture, and surface of an approaching obstack, unlike sonar and infrared sensor systems. Capacitive sensors are inherently simple, consisting of two conductive plates with minimal supporting electronics located near th. sensing site. This greatly increases sensor reliability, which is an important consideration in: hazardous environments with extremes of temperature, radiation, and corrosives.

Capacitance-based proximity sersors may be divided into two classes based on whether u: not the obstacle forms one plate of capacitive sensor. The most common capacitive sensors consist of a single plate on the sensor itself and use the obstacle as the second capacitor plate. This configuration works well in environments in which obstacles are nearby. conductive and electrically grounded [2]. However, parasitic capacitances to ground are problematic and require the use of driven-guard shielding techniques which add to the complexity of the electronics located near the sensor.

The WHole Arm Proximity (WHAP) sensor described in this paper falls into the second class of capacitive sensors. The WHAP sensor uses two plates on a single substrate to generate and measure changes in an electric field. Conductive or dielectric obstacles disturb the electric field through a shielding effect and alter the measured capatcitance. Since the electric field between the two plates is well-defined by the conductor atrangement, it is possible to reconstruct the obstacle surface and range more accurately. No active shielding is required, and this type of sensor is insensitive to the electrical potential of the obstacle. This feature becomes particularly important in outdoor or space environments without the ubiquitous capacitive coupling to the power distribution system.

In this paper, pre!iminary esults of experiments with the WHAP sensur are presented. First. a 2D finite element moder is developed to atd in designing sensors to meet specitiv: specitications. Details of calculating mutual capacitances among multiple conductors are 
provided. Experimental data from four different sensor designs is then compared to the model predictions. Finally, eight sensor elements are used on a two-link robot t.) demonstrate a straightforward technique for avoiding collisions with conductive and nonconductive obstacles such as metal poles and concrete.

\section{Theory}

A schematic 2D model of the WHAP sensor is given in Figure 1 . Electrode 1 is driven by an oscillator, while electrode 2 is connecied to an amplifier for sensing capacitor charge. For a fixed oscillator drive voltage, this charge output signai, $V_{c}$, is proportional to the sensor capacitance. The sensor capacitance is altered by the presence of obstacles within the electric field. Under the assumption that the wavelength of the oscillator is much larger than the dimensions of the sensor, the analysis becomes an electrostatic problem containing two conductive electrodes and obstacles of unknown composition. In this paper only conductive obstacles will be considered in the sensor analyses, although nonconductive obstacles can be sensed and will be considered in future work.

The obstacle is assumed to be a conductive plate oriented parallel to the sensor ( 3 in Figure 1). This assumption is reasonable for collision avoidance purposes if the WHAP sensor is much smaller than the obstacle and the obstacle is relative!y far away. This model with the three capacitances and one impedance of concern is given in Figure 1. The charge amplifier configuration of the WHAP circuitry (discussed later) senses changes only in $C_{12}$, so the problem consists of relating the obstacle geometry to this capacitance. In the present paper, we will use finite element analyses (FEA) to calculate the variation of $C_{t 2}$ with distance. $d$, for four different sensor geometries. Analytic expressions for calculating $C_{t, 3}$ and $C_{2}$; were developed by Getsinger [7] and Cohn [8], and an approximate expression for $C_{I_{2}}$ was developed by Novak [9]. However, these expressions are useful only for flat, conductive. and grounded $\left(Z_{g_{n d}}=0\right)$ obstacles. The FEA technique described here will permit more general analysis of sensor response to nonconductive or poorly grounded obstacles, as well as obstacles of arbitrary shapes.

Capacitances in the model may be calculated from electric field intensities. For the twodimensional system, the charge, $Q$, on a conductor may be written as

$$
Q=\oint_{S} \varepsilon E \cdot d S
$$

where $E$ is the electric field vector, and $S$ is a surface completely enchusing the conducurs. In the 2D model considered here, the surface $S$ reduces to a continumus pith around the conductor. 
The electric field intensities in the model were calculated using FEA techniques. The WHAP sensor was modeled by defining a geometry and creating a mesh of nodes which subdivide the geometry into elements. Matrix equations for the voltages at the nodes can be generated based upon the geometry, boundary conditions (voltages on conductors), and material properties (conductivity and dielectric constant). Electric field intensities at each node are then calculated from these voltages. An integration path cutting through all the field lines emanating from a conductor was specified, and the charge calculated using a discretized version of (1).

The charge on each conductor is affected by both of the other conductors presen, in the electrostatic field, however. Only a portion of the charge on electrodes 1 and 2 is due to the mutual capacitance, $C_{t 2}$, between electrodes 1 and 2 . The component of charge on electrodes 1 and 2 due to only $C_{12}$ may be calculated using the general relationship between the charges and voltages of conductors in an electrostatic field [10] of the form

$$
\left[\begin{array}{l}
Q_{1} \\
Q_{2} \\
Q_{3}
\end{array}\right]=\left[\begin{array}{lll}
c_{11} & c_{12} & c_{13} \\
c_{21} & c_{22} & c_{23} \\
c_{31} & c_{32} & c_{33}
\end{array}\right] \times\left[\begin{array}{c}
V_{1} \\
V_{2} \\
V_{3}
\end{array}\right],
$$

where $Q$ and $V$ are a $1 \times 3$ vectors of the charges and potentials of the three conductors, $c_{i}$ is defined as the capacity of conductor $i$, and $c_{i, j} i \neq j$, are the coefficients of induction. The sapacity and the coefficients of induction are functions of the size, shape, and position of the conductors and the dielectric properties of the intervening space. The charge on a given conductor is thus a scaled, linear combination of all conductor voltages. Furthermore, the mutual capacitance between conductors 1 and 2 , is related to the coefficient of induction through

$$
C_{12}=-c_{12}=-c_{21} .
$$

At this point one may obsere that if the FEA simulations are performed by specifying houndary condition of $V=\left[\begin{array}{lll}1 & 0 & 0\end{array}\right]^{\mathrm{T}}$. the mutual capacitance of the WHAP sensor mat be determined by negating the charge on conductor 2. This technique wats used for generating the results in the following section.

\section{Moleling Results}

The data below were calculated using $\operatorname{COSMOS}^{2} / \mathrm{M}$ v 1.61 finite element andlysis software [SRAC. Santa Monicat, CA. USA] ruming on a Sun t/301) workstation. A twis-dimensiomal 
model of a WHAP sensor was developed and evaluated for various electrode spacing and obstacle heights.

Four different sensor geometries were simulated. The electrode width, w, was fixed at 15 $\mathrm{mm}$ while the gap, $s$, was set to $3,38,73$, and $108 \mathrm{~mm}$, respectively. The distance, $d$, from the two sensor electrodes to the conductive workpiece was varied from 10 to $600 \mathrm{~mm}$.

The accuracy of the model was verified by comparing the simulation results for the noobstacle situation (distance $=\infty$ ) with the capacitance value, $C_{N}$, given by Binns [11] as

$$
C_{N}=\frac{K(k)}{K^{\prime}(k)} \quad \text { where } \quad k=\frac{s}{2 w+s}
$$

and $\mathrm{K}$ is the complete elliptic integral of the first kind and $\mathrm{K}^{\prime}$ is its complement.

These results for the no-obstacle case are tabulated in Table I. The same mesh was usew for all four sensor simulations; thus the modeling error increases with decreasing gap size, due to the higher voltage gradients and a correspondingly inaccurate mesh.

Table I. No obstacle two-dimensional WHAP capacitances

Sensor electrode width $=15 \mathrm{~mm}$

\begin{tabular}{|l|l|l|l|}
\hline Gap(mm) & Binns(Eq.4) & FEA & $\%$ Dift. \\
\hline 3 & $1.24 \epsilon_{0}$ & $1.32 \epsilon_{0}$ & 6.5 \\
\hline 38 & $0.60 \epsilon_{0}$ & $0.63 \epsilon_{0}$ & 5.0 \\
\hline 73 & $0.50 \epsilon_{\circ}$ & $0.52 \epsilon_{0}$ & 4.0 \\
\hline 108 & $0.45 \epsilon_{0}$ & $0.46 \epsilon_{0}$ & 2.2 \\
\hline
\end{tabular}

The simulation results are plotted in Figure 2. All four curves rise asymptotically toward the value given in Table I above. In addition, for distances greater than about $125 \mathrm{~mm}$. the slopes of all four curves are approximately equal. This suggests that gap variations will not significantly affect the long-distance obstacke sensitivity.

The curves corresponding to the smaller gaps, 3 and $38 \mathrm{~mm}$, exhithit a marked increase in slope as the obstacle comes nearer. This effect is most pronounced for obstacte distances less than about $125 \mathrm{~mm}$. This increase in sensitivity of the 'WHAP sensor for nearby 
obstacles is used later to enhance avoidance trajectory perturbattins fior preventing imminent collisions.

\section{Experimental Methods}

The prototype WHAP sensor was fabricated using a three layer printed circuit board. The bottom layer was used for power and drive signals. The top layer contained the charge amplifier components (an op-amp, one resistor, and one capacitor as in Figure 1) and the middle layer was grounded for isolation. The 15 by $8 \mathrm{~mm}$ sensor pads were arranged as in Figure 3. Only the charge amplifier integrated circuits (LF347) needed to be on the sensor board. As mentioned earlier, no driven guard is necessary in this design since the charge amplifier configuration is insensitive to parasitic capacitances to ground. By varying the connections to the driving oscillators and charge amplifiers, it was possible to vary the effective spacing of the sensor electrodes to achieve arrays of either eight sensors with $3 \mathrm{~mm}$ gaps or four sensors with gaps of $3,38,74$, and $108 \mathrm{~mm}$. The connections for eight $3 \mathrm{~mm}$ gap sensors are given in Figure 3. For the robot application described below, two eightsensor arrays were used (one on each link) but data was only acquired from every other sensor. The electical connections were varied to create the four sensors with different geometries discussed below.

Two frequencies were used to efficiently encode data from two sensors onto a single signal channel through a charge amplifier (top of Figure 3). The output signal from charge amplifier 0 consisted of the sum of sinusoids at $f_{1}$ and $f_{2}$. In these experiments $f_{1}=100 \mathrm{kHz}$ and $f_{2}=153 \mathrm{kHz}$, although these values are not critical. The $f_{1}$ component was amplitude modulated by obstacles in the electric field in the leftmost gap. The $\mathrm{f}_{2}$ component was similarly modulated by changes in the electric field in the neighboring gap. This pattern repeats for the remaining sensors.

Synchronous detection circuitry was used to measure the amplitude of the corresponding frequency component in the charge amplitier output. These circuits provided an extremely low noise signal output by phase- and frequency-locking onto the input drive signal. Because of this, no shielding was required on the signal leads. Only the cables leading to the drive electrodes must be shielded to minimize parasitic coupling to the charge amplifier inputs.

A large fraction of the output signal from the synchronous detector was due to the electric field within the dielectric substrate of the printed circuit board between sensor electrodes. A DC offset was subtracted from this signal prior to the final stage of amplification. The resulting analog signal was digitized using a 12-bit A/D converter.

\section{DISCLAIMER}

\footnotetext{
This report was prepared as an account of work sponsored by an agency of the United States Government. Neither the United States Government nor any agency thereof, nor any of their employees, makes any warranty, express or implied, or assumes any legal liability or responsibility for the accuracy, completeness, or usefulness of any information, apparatus, product, or process disclosed, or represents that its use would not infringe privately owned rights. Reference herein to any specific commercial product, process, or service by trade name, trademark. manufacturer, or otherwise does not necessarily constitute or imply its endorsement, recommendation, or favoring by the United States Government or any agency thereof. The views and opinions of authors expressed herein do not necessarily state or reflect those of the United States Government or any agency thereof.
} 


\section{Experimental Results}

The overall gain of the synchronous detection circuitry was $80 \mathrm{~V} / \mathrm{V}$. The output of the electronics may be related to the WHAP capacitance, $C_{12}$, by

$$
V_{o}=\frac{C_{12}+C_{p c b}}{C_{f}} \times 80.0 \text {, }
$$

where $C_{p<b}$ is the capacitance due to the electric field within the printed circuit board and connecting leads, and $C_{f}$ is the feedback capacitance of the charge amplifier (Figure 1 ). The feedback resistor, $R_{f}$, was chosen to prevent saturation, and yet not affect the response at the driving frequency. The output noise was about $14 \mathrm{mV}$ with $C_{f}=2.2 \mathrm{pF}$ and $V_{i n}=10 \mathrm{~V}$, resulting in an equivalent noise capacitance of $0.04 \mathrm{fF}$. The nutput signal was stable and drifted no more than $5 \mathrm{mV}$ over a 4 hour stability test.

The $C_{p c b}$ term in (5) provided a large offset to the output voltage. A DC offset adjustment circuit was used to prevent $A / D$ saturation for the large variations in output signal from the sensor with the $3 \mathrm{~mm}$ gap. Since only relative changes in capacitance versus output position were significant in these experiments, no attempt was made to measure capacitances absolutely.

Outputs from the four differently-sized sensors on the single substrate were recorded simultaneously as the substrate was moved in $0.2 \mathrm{~mm}$ increments away from a 400 by $480 \mathrm{~mm}$ aluminum obstacle. This experiment was repeated five times and the results averaged to give the data in Figure 4. These curves may be considered to be the sensor response functions for the particular case of a flat, conductive obstacle. The sensor response was quite repeatable and stable; the maximum standard deviation over all 2400 data points in the five trials was 4.9 A/D units. Error bars would be barely visible in Figure 4.

The signals from the WHAP sensor with the $3 \mathrm{~mm}$ gap varied greatly over the first $50 \mathrm{~mm}$ of travel and then levelled off. As the gap between electrodes increased. the overall dynamic range of the output signal decreased, especially for sensors with 3 and $38 \mathrm{~mm}$ gaps (note the change in scale in Figure 4). This effect was also observed in the 2D simulation (Figure 2). The two sensors with the smallest gaps $(3$ and $38 \mathrm{~mm}$ ) p-ribited transition regions or "corners" between near and far obstacles. This effect was also observed, althougin somewhat less pronounced, in Figure 2.

For distances greater than the "corners", all four sensor signals increased similarly with decreasing slopes until the output was indistinguishable from the background noise. This point was about $400 \mathrm{~mm}$ for all four sensors. This distance was considered to be the maximum working distance for this particular sensor geometry. As predicted by the 2D 
model, the maximum working distance is not a function of the sensor gap size, given at fixed electrode size.

The sensors with smaller gaps offer superior sensitivity at closer working distances, given similar background noise levels. For example, the sensor with the $3 \mathrm{~mm}$ gap would provide much more sensitivity (or less position uncertainty) at distances less than about $50 \mathrm{~mm}$ than any of the other sensors. However, sensors with larger gaps may offer advantages when sensing objects with smaller profiles. This hypothesis was not tested but seems reasonable considering the larger extent of the electric field.

Experiments were performed using a 350 by $350 \mathrm{~mm}$ concrete block to investigate the WHAP sensor response to dielectric materials. The shape of the sensor output versus distance curves were similar, indicating the action of a shielding effect related to that observed for conductive obstacles. The overall signal variation for distances from about $5 \mathrm{~mm}$ to $400 \mathrm{~mm}$ was about a factor of four smaller, however. By using sufficiently large gains in the obstacle avoidance system described next, the same control was used to avoid both a conductive post and a concrete block.

Interestingly, as the obstacle came very close to the sensor $(<.5 \mathrm{~mm})$, the sensor capacitance rose rapidly, in opposition to the phenomenon observed for conductive obstacles. This effect has been described before (13) and is due to the replacement of a poor dielectric (air) by a better dielectric (concrete) in the sensor electric field. The following experiments were designed to avoid this operating region of the sensor. Following a more detailed analysis of this behavior, future control systems will be designed to permit operation in this regime.

\section{Application to a Two Link Robotic Arm}

Four sensors with $3 \mathrm{~mm}$ spacing on each of two WHAP sensor arrays were used on a two link planar robotic arm as shown in Figure 5. In these preliminary experiments, obstacles were placed only on one side of the arm; therefore. both sensor arrays were on the same side of the two links. Both conductive and dielectric obstacles were placed in the robot workspace along the desired trajectory of the arm. A trajectory generator provided a smooth motion along the desired path while an obstacle avoidanre loop provided a perturbation away from obstacles which were within the WHAP sensor's range (Figure 6). Since the WHAP sensor's output is dependent on the distance from an obstacle, these measurements can be transformed into the changes in robot joint angles required on avoid the obstacle.

Based on the analysis of the previous section, some assumptions were made regarding the obstacle's shape and position in the 2D plane. Keleally, the sensor output is given as 


$$
z_{i j}=h_{i j}\left(x_{i j}-x_{o b}, \gamma_{o b}\right)
$$

where $z_{i j}$ is the output of sensor $j$ on link $i, x_{i j}$ is the $(x, y)$ position of sensor $i j$ in world coordinates, $x_{a b}$ is the $(x, y)$ position of the obstacle in world coordinates, $\gamma_{a b}$ are parameters related to the obstacle's shape, and $h_{i j}$ is the sensor's response function. In the previous section, the sensor's response function was plotted for a single obstacle shape (a large plate) which was perpendicular to the sensor. Since the shape parameters for the objects are not known, it was assumed that all obstacles are large plates perpendicular to the sensor. This is a good approximation in most cases, since the obstacles are larger than the sensor electrodes. Now, the previous equation simplifies to

$$
z_{i j}=h_{i j}\left(y_{i j, o b}\right)
$$

where $y_{i j, o b}$ is the perpendicular distance between sensor $i j$ and the obstacle. As shown in the previous section, the WHAP sensor's output is related nonlinearly to the distance from the object. If the control is to respond linearly to the distance from the object, the expression for $h_{i j}$ would have to be inverted in order to determine $y_{i j, o b}$ from $z_{i j}$. However, the shape of the nonlinear relationship is ideal for the task of obstacle avoidance since the sensor output versus distance is inherently a useful potential function. As the sensor moves closer to the obstacle, the output decreases dramatically (Figure 4, $3 \mathrm{~mm}$ gap). This potential function is used directly in the current control structure by perturbing the system based upon the error between the desired sensor output (when no obstacles are present) and the actual measurement.

In order to move away from obstacles, differential changes in $(x, y)$ position with respect to each sensor must be transformed into differential changes in the robot joint angles. For sensor $1 j$ on link 1 , the differential relationship with respect to the coordinate frame of sensor $1 j$ is [12]

$$
\left[\begin{array}{r}
{ }^{1} \dot{x} \\
{ }^{1} \dot{y}
\end{array}\right]-{ }^{1 j} J\left[\begin{array}{l}
\dot{\theta}_{1} \\
\dot{\theta}_{2}
\end{array}\right] \text { where }{ }^{1} J J=\left[\begin{array}{cc}
0 & 0 \\
d_{1} & 0
\end{array}\right]
$$

${ }^{\prime \prime} x_{x}$ and ${ }^{\prime} y_{j}$ are the $x, y$ velocities of sensor $l j$ with respect to it's own coordinate frame. $\dot{\theta}_{t}$ and $\dot{\theta}_{2}$ are the joint angle velocities, ${ }^{\prime} J$ is the Jacobian with respect to the condinate trame $1 j$, and $d_{l j}$ is the distance of sensor $1 j$ from joint 1 . For sensor $2 j$ on link 2, the differential relationship with respect to the coordinate frame of sensor $2 j$ is 


$$
\left[\begin{array}{c}
2 j \dot{x} \\
2 j \dot{y}
\end{array}\right]={ }^{2 j}\left\{\begin{array}{l}
\dot{\theta}_{1} \\
\dot{\theta}_{2}
\end{array}\right] \text { where } \quad 2 j J=\left[\begin{array}{cc}
d_{2 j} \sin \theta_{2} & 0 \\
\left(d_{1} \cos \theta_{2}+d_{2 j}\right) & d_{2 j}
\end{array}\right] \text {, }
$$

${ }^{2 j} \dot{x}$ and ${ }^{2 j} \dot{y}$ are the $x, y$ velocities of sensor $2 j$ with respect to it's own coordinate frame, ${ }^{2 j} J$ is the Jacobian with respect to the coordinate frame $2 j$, and $d_{2 j}$ is the distance of sensor $2 j$ from joint $2, d_{i}$ is the length of link 1 . Given the previous assumption regarding the obstacle being perpendicular to the sensor, only the $y$ romponent of these equations is observable.

The Jacobian pseudo inverse used in Figure 6 to transform sensor errors to changes in robot joint angles depends on the sensors available. For the case of a single WHAP sensor on link 2 , the differential transformation is

$$
\Delta^{2 j} y=\left[\left(d_{1} \cos \theta_{2}+d_{2 j}\right) d_{2 j}\left[\begin{array}{c}
\Delta \theta_{1} \\
\Delta \theta_{2}
\end{array}\right]\right.
$$

This is an underdetermined system, meaning there are infinitely many solutions given by

$$
\Delta \theta_{2}=\frac{1}{d_{2 j}}\left[\Delta^{2 j} y-\left(d_{1} \cos \theta_{2}+d_{2 j}\right) \Delta \theta_{1}\right]
$$

where $\Delta \theta_{l}$ can be any number. For the case of a single WHAP sensor on both link 1 and link 2 , the system is uniquely determined system with solution

$$
\left[\begin{array}{l}
\Delta \theta_{1} \\
\Delta \theta_{2}
\end{array}\right]=\left[\begin{array}{cc}
\frac{1}{d_{1 j}} & 0 \\
-\frac{\left(d_{1} \cos \theta_{2}+d_{2 j}\right)}{d_{1 j} d_{2 j}} & \frac{1}{d_{2 j}}
\end{array}\right]\left[\begin{array}{l}
\Delta^{1 j} y \\
\Delta^{2 j} y
\end{array}\right]
$$

Notice that if $\Delta^{l j} y=0$ then the solution is simply $\Delta \theta_{2}=\Delta^{2 j} y / d_{2 j}$, which is the same as the underdetermined case when $\Delta \theta_{2}$ is chosen to be zero. Also notice that if $\Delta^{2 j}=0$ the perturbation from sensor $l j$ will cause joint 2 to move in a direction which counters joint l's motion. This motion is required in order for the perpendicular velocity at sensor $2 j$ (1) be 
zero. This effect was found to be undesirable and was removed by letting the off diagrmal term equal zero. This is equivalent to allowing sensor $2 j$ have a positioning error of

$$
\text { error }=\frac{-\left(d_{1} \cos \theta_{2}+d_{2 j}\right)}{d_{1} d_{2 j}} \Delta \theta_{1} \text {, }
$$

when link 1 encounters an obstacle.

For the case of $m$ sensors on link 1 and $n$ sensors on link 2, the system is overdetermined. The least squares solution is given by

$$
\left[\begin{array}{c}
\Delta \theta_{1} \\
\Delta \theta_{2}
\end{array}\right]=J \cdot\left[\begin{array}{c}
\Delta^{11} y \\
\vdots \\
\Delta^{1 m} y \\
\Delta^{21} y \\
\vdots \\
\Delta^{2 n y}
\end{array}\right],
$$

where $J^{+}$is the Moore-Penrose pseudo inverse

$$
J^{+}=\left(J^{T}\right)^{-1} J^{T} \quad \text { where } \quad J=\left[\begin{array}{cc}
d_{11} & 0 \\
\vdots & \vdots \\
d_{1 m} & 0 \\
\left(d_{1} \cos \theta_{2}+d_{21}\right) & d_{21} \\
\vdots & \vdots \\
\left(d_{1} \cos \theta_{2}+d_{2 n}\right) & d_{2 n}
\end{array}\right] \text {. }
$$

Again, when the signals from the link 2 sensor array are zero and those from link 1 sensor array are nonzero, joint 2 will move opposite joint 1 because of the coupling terms. This effect was removed by setting the $\left(d_{1} c_{2}+d_{2 j}\right)$ terms to zero. The resulting least squares solution is 


$$
\Delta \theta_{1}=\frac{\sum_{i=1}^{m} d_{1 i} \Delta^{1 j} y}{\sum_{i=1}^{m} d_{1 i}^{2}} \text { and } \Delta \theta_{2}=\frac{\sum_{i=1}^{n} d_{2 i} \Delta^{2 j} y}{\sum_{i=1}^{n} d_{2 i}^{2}}
$$

These equations, with $m=4$ and $n=4$, were used together with experimentally determined feedback gains in the experiments discussed below.

The experimental results of the two link robot avoiding three obstacles are shown in Figures 7-9. Figure 7 shows the tip positicn of the robot as link 2 avoids a $40 \mathrm{~mm}$ diameter metal pipe and a 120 by $190 \mathrm{~mm}$ concrete block. Near the end of the trajectory, the robot is stopped by a 100 by $100 \mathrm{~mm}$ metal plate blocking the path of link 1 . In Figure 8 , the two large perturbations in the joint 2 path are caused by the pipe and the block. The smaller variations from the desired position are joint level PD coritrol errors resulting from the motion of joint 1 . The tip of the arm is moving at $0.9 \mathrm{~m} / \mathrm{s}$ when it encounters these obstacles. In Figure 9, the large perturbation at the end of the trajectory is caused by the metal plate. The smaller offsets during the 1 to $9 \mathrm{~s}$ interval are the tracking errors of the joint level PD controller. Since this control scheme only perturbs the desired trajectory, it is possible to eventually build up enough joint position error to overcome the perturbation from the WHAP sensors. When this occurs, the trajectory generator will need to be undated and a new path must be planned.

\section{Summary}

This paper presents preliminary experiments usifig a capacitive proximity sensor for use in collision avoidance. The WHAP sensor comprises both plates of the capacitor, permitting better characterization of the extent of the electric field and thus the spatial sensitivity of the device. The sensor output changes instantaneously for conductive obstacles from 0 to $400 \mathrm{~mm}$ away. Data for dielectric obstacles, not discussed explicitly in this paper, demonstrate that the sensor responds differently to conductive and dielectric obstacles, in a manner similar to the examples in [13]. A 2D finite element model, developed as a design tool for the sensor, accurately predicts trends in the sensor response to large conductive obstacles. Straightforward control algorithms were used with WHAP sensors mounted on a two link planar robotic arm. The robot avoided colliding with metal and concrete obstacles while maintaining a high tip velocity. 


\section{Future Work}

Future work with the WHAP sensor will involve additional refinement of the sensor itself, as well as further development of more complex collision avoidance algorithms. More elaborate sensor/obstacle models will be examined to determine the effects of obstacle geometry and composition on the spatial sensitivity of different sensor designs.

WHAP sensors will be mounted on a 6 DOF robot, mandating the use of path planning techniques such as those of Lumelsky and Cheung [4], Krough [6], and Tilove [5]. Future sensors will be fabricated on flexible printed circuit boards to conform to, and provide collision protection for, most robot link surfaces.

\section{Acknowledgement}

The direction for this research was shaped by early, often lively, discussions with Jamie Wiczer. We also wish to thank Bob McNeely for fabricating the prototype sensors and analog signal conditioning boards, Jon Bryan for his efforts in designing and fabricating the data acquisition system, and Jim Akins for keeping the workstation and robot operating.

\section{References}

[1] E. Cheung and V. Lumelsky, "Development of Sensitive Skin for a 3D Robot Arm Operating in an Uncertain Environment," Proceedings of the 1989 IEEE International Conference on Robotics and Automation, Scottsdale, AZ, May 14-19, 1989, pp. 1056-1061.

[2] J. M. Vranish and D. S. Chauhan, "Tri-mode collision avoidance skin for robot arms in space," Proceedings of the Third International Symposium on Robotics and Manufacturing: Research, Education, and Applications, Burnaby, British Columbia, CANADA, July 18-20, 1990, pp. 189-195.

[3] E. Cheung and V. Lumelsky, "Motion planning for robot arm manipulators with proximity sensing," Proceedings of the 1988 IEEE International Conference on Robotics and Automation, Philadelphia, PA, April 24-29, 1988, pp. 74()-745.

[4] V. Lumelsky and E. Cheung. "Toward a sate real-tirne robot teleoperation: Automatic whole-sensitive arm collision avoidance trees the operator for global control," Proceedings: of the 1991 IEEE International Conterence on Robotics and Automation. Sacramento, C.A. $1991, \mathrm{pP} .797-802$.

[5] R. B. Tilove. "Local obstacle avoidance for mobile robots based upon the method of artificial potentials." Proceedings of the 1990 IEEE International Conference on Robotic: and Automation, Cincinnati. OH. May 13-18. 194), pp. 56x-571. 
[6] B. H. Krough, "A generalized potential field approach to obstacle avoidance control," Robotics Research: The next five years and beyond," SME Conference Proceedings, August. 1984.

[7] W. J. Getsinger, "Coupled rectangular bars between parallel plates," IRE Trans. Microwave Theory and Techniques, pp. 65-72, 1962.

[8] S. B. Cohn, "Shielded coupled-strip transmission line," IRE Trans. Microwave Theory and Techniques, pp. 29-38, 1955.

[9] J. L. Novak and J. J. Wiczer, "A high resolution capacitive imaging sensor for manufacturing applications," Proceedings of the 1991 IEEE International Conference on Robotics and Automation, Sacramento, CA, 1991, pp. 2071-2078.

[10] J. C. Maxwell, A Treatise on Electricity and Magnetism, Third Edition, Oxford University Press, London, 1946.

[11] K. J. Binns and P. J. Lawrenson, Analysis and Computation of Electric and Magnetic Field Problems, Pergammon Press, New York, 1973.

[12] R. P. Paul, Robot Manipulators: Mathematics, Programming, and Control, The MIT Press, Cambridge, Massachusetts, 1981.

[13] D. Lawrence, "Proximity sensors that can take the heat," Sensors, December, 1986, pp. $37-39$. 


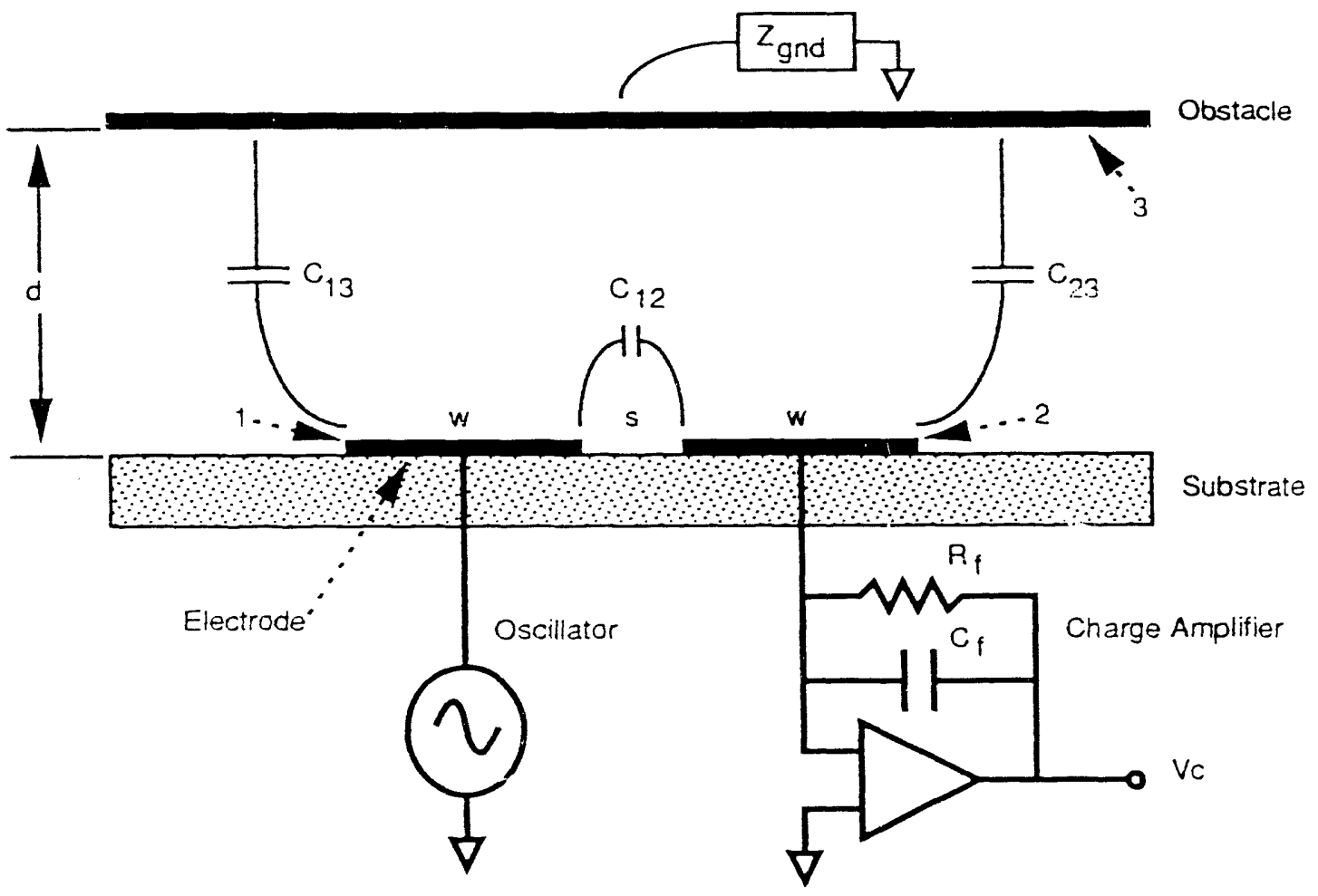

Figure 1. WHAP Geometry and Lumped Component Electrical Model 


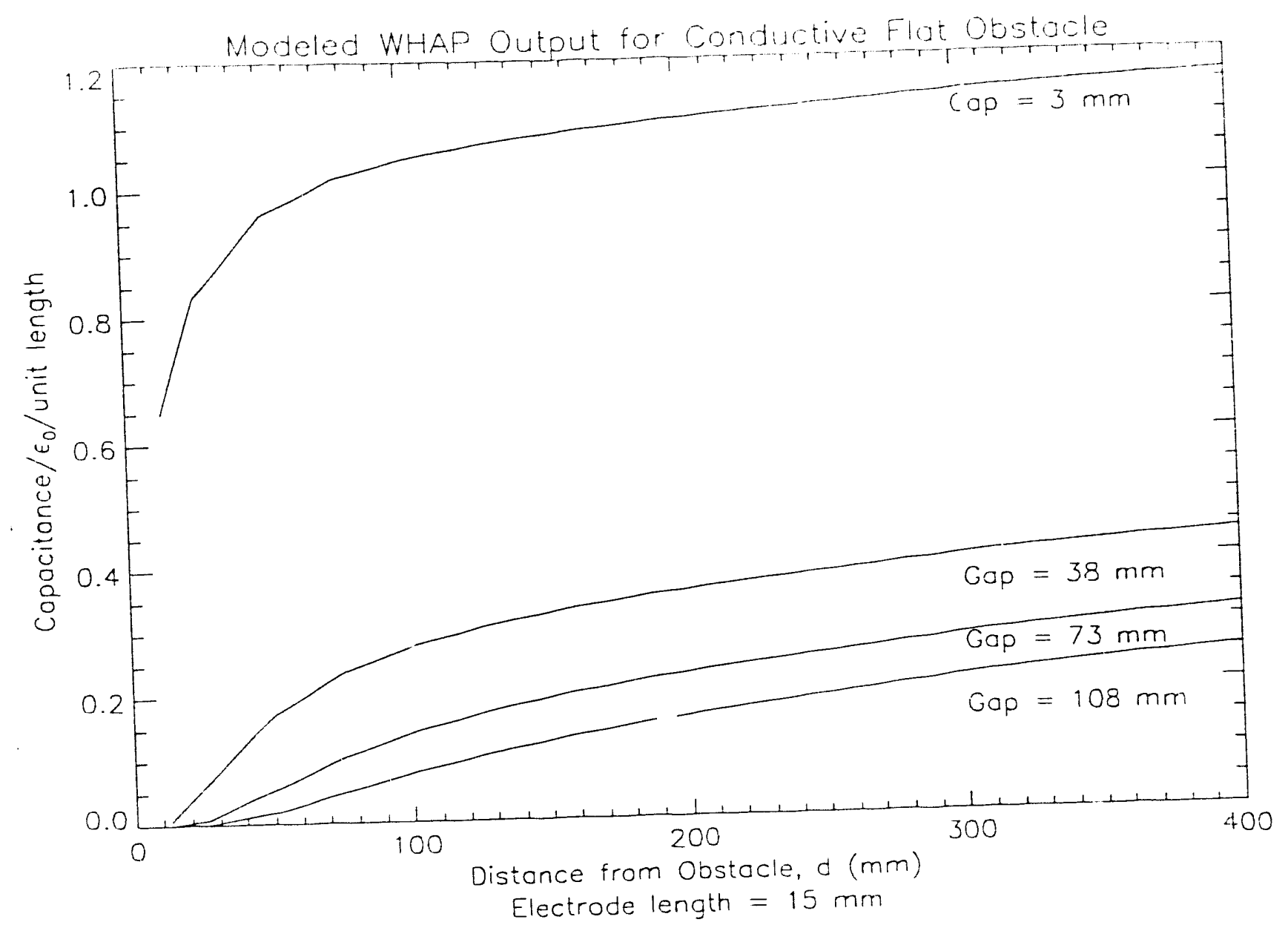

Figure 2. Modelled WHAP Output Versus Distance for Four Different Sensor Geometries 
$\mathrm{CA}=$ Charge Amplifier circuit

SD1 = Synchronous Detection circuit for frequency $\mathrm{f} 1$

SD2 = Synchronous Detection circuit for frequency 12

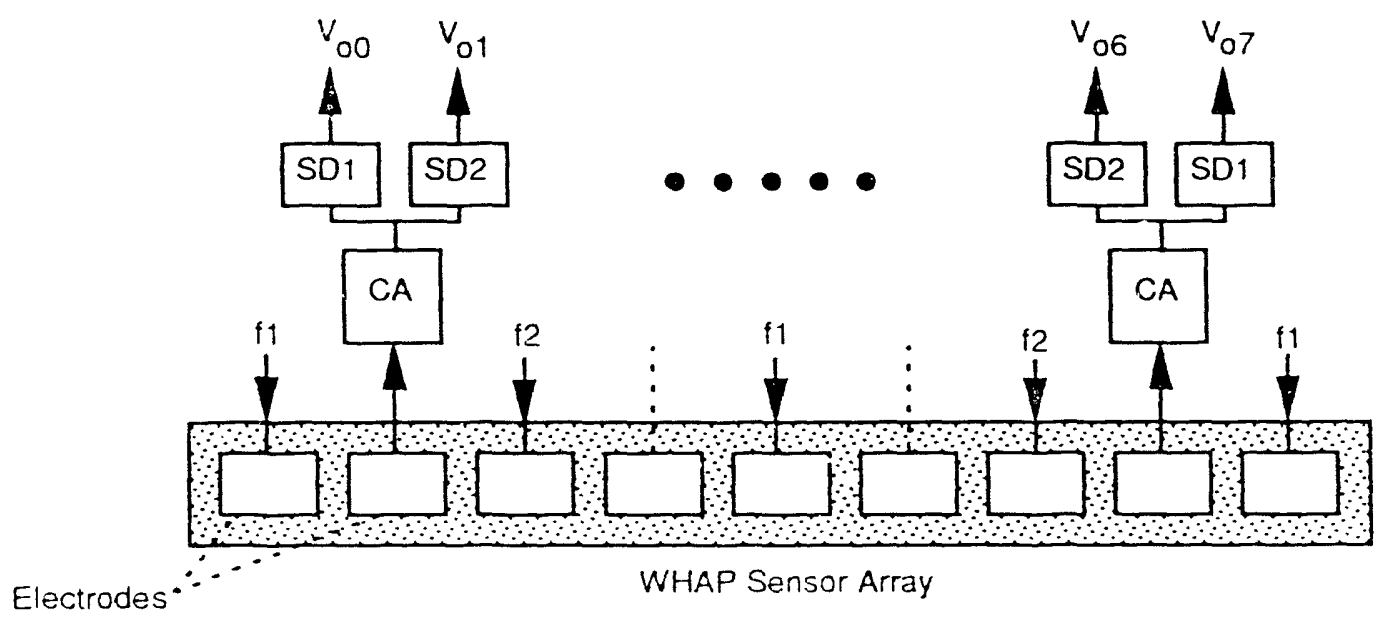

Figure 3. WHAP Sensor Array Geometry and Electrical Connections 


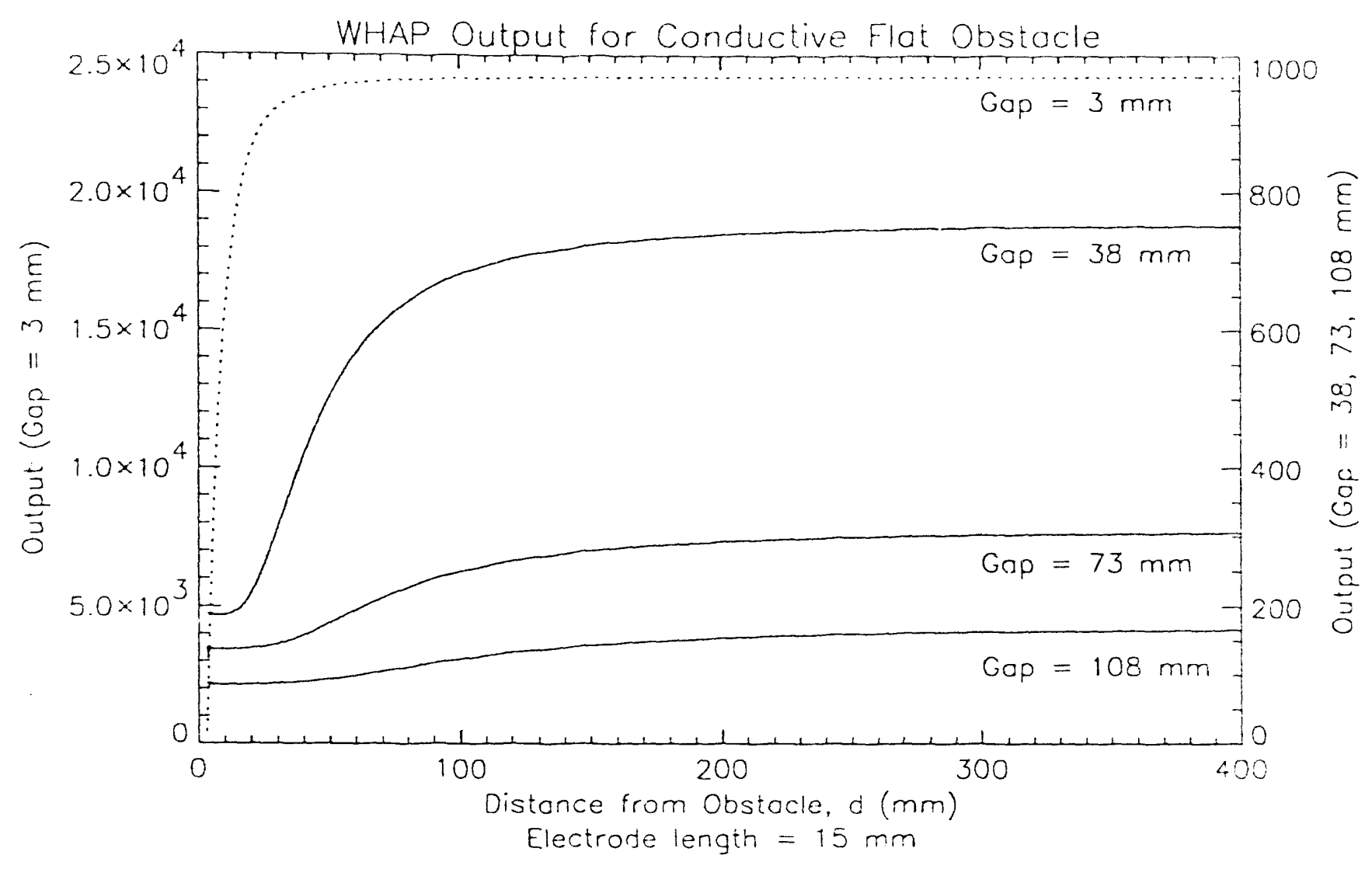

Figure 4. WHAP Output Versus Distance for Four Different Sensor Geometries 


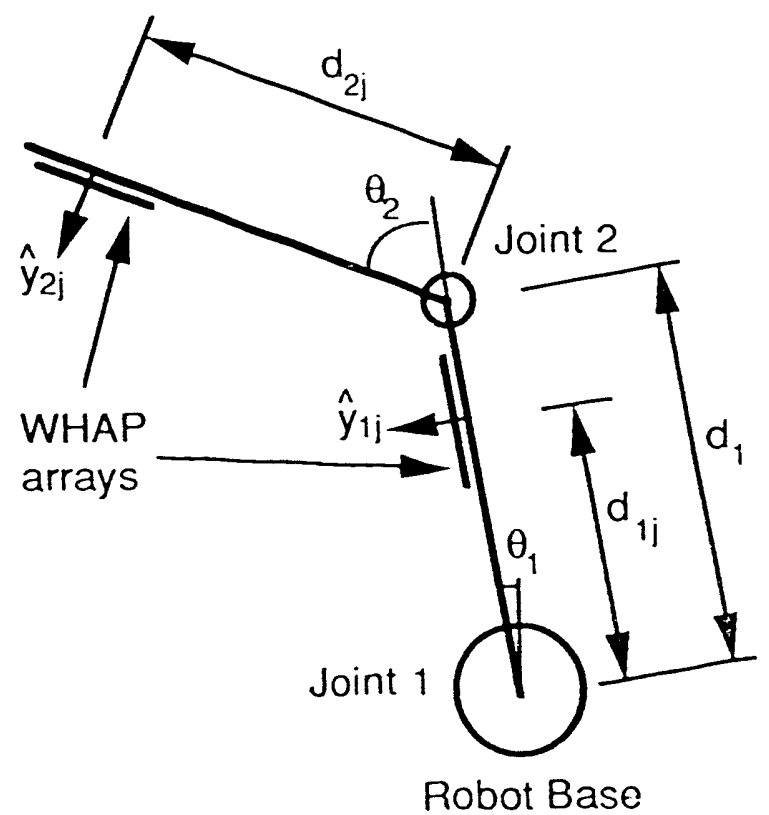

Figure 5. Two Link Planar Arm with WHAP sensors.

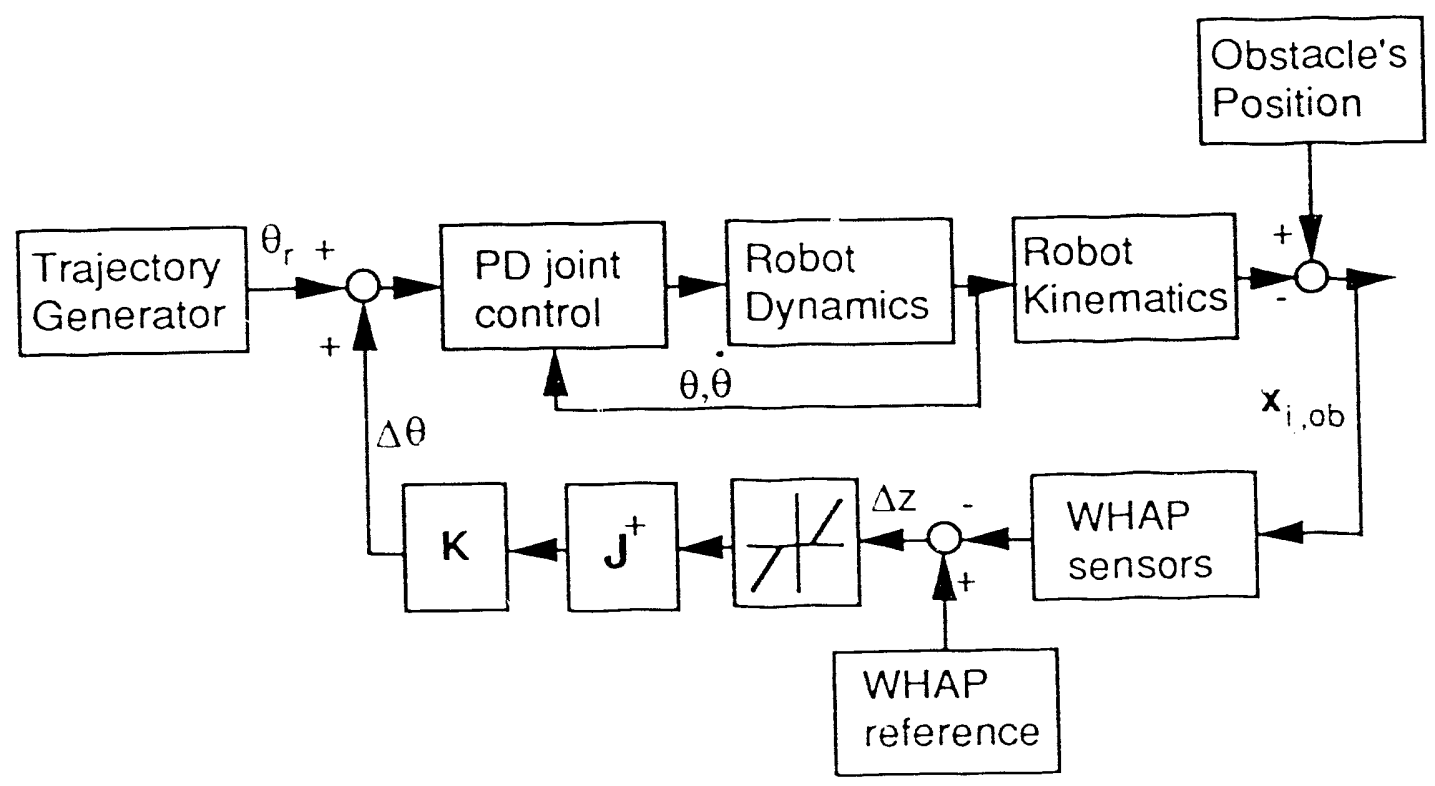

Figure 6. Control Structure for Obstacle Avoidance. 


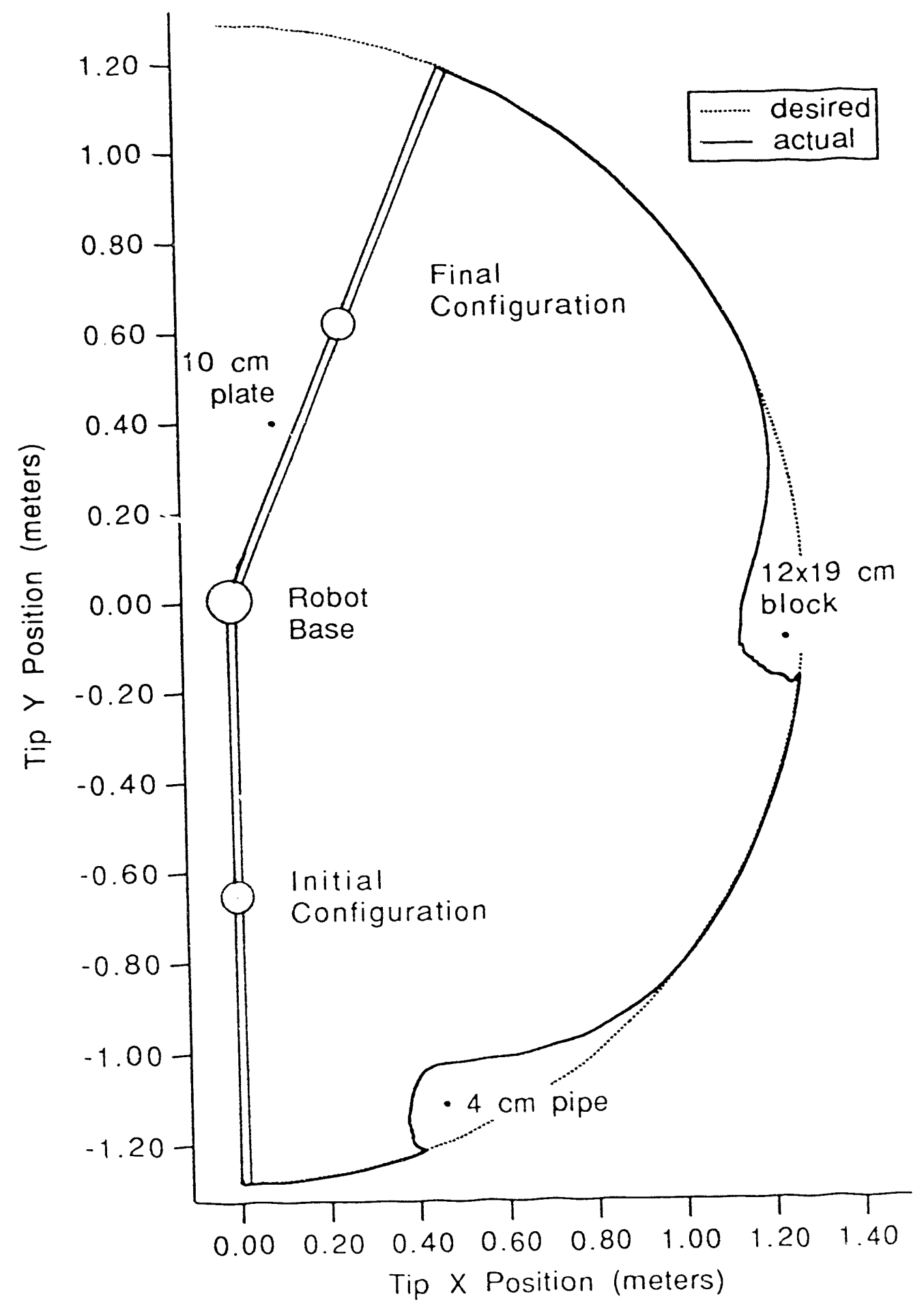

Figure 7. Tip position of the two link robotic arm during obstacle avoidance experiment. 


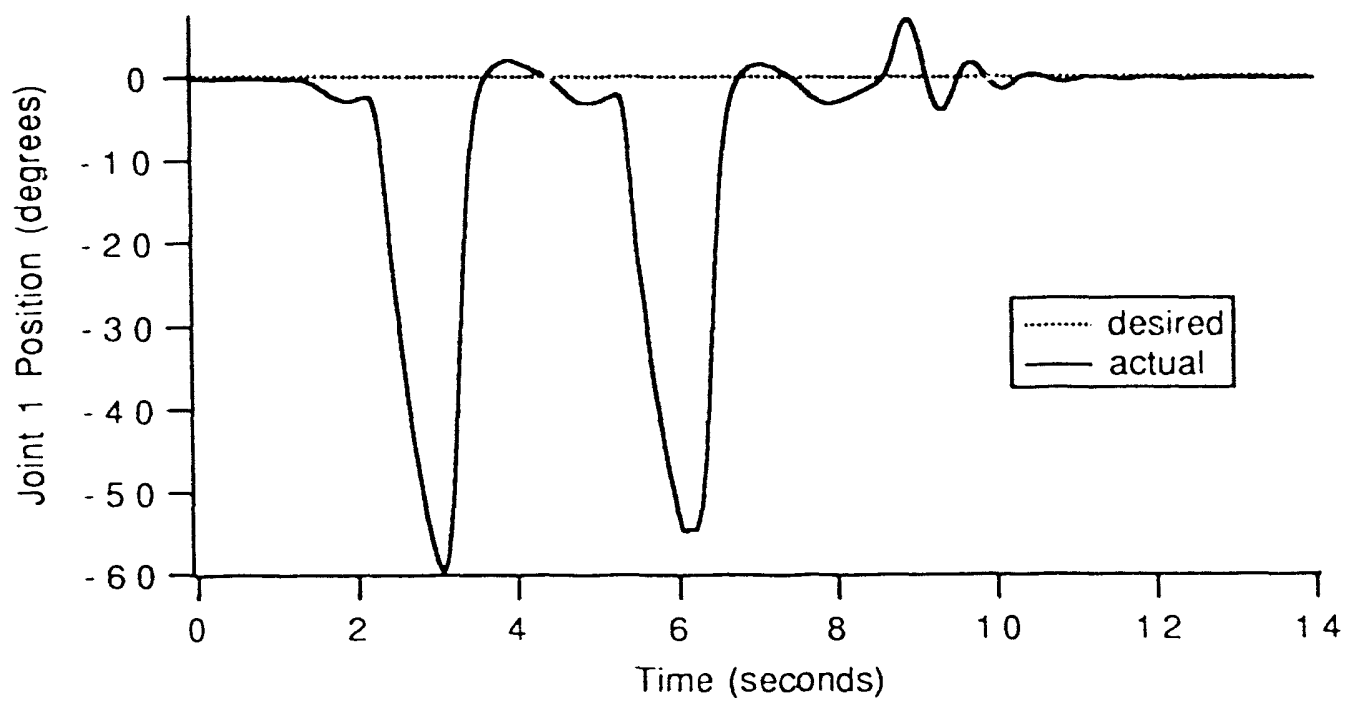

Figure 8. Position of joint 2 during the obstacle avoidance experiment.

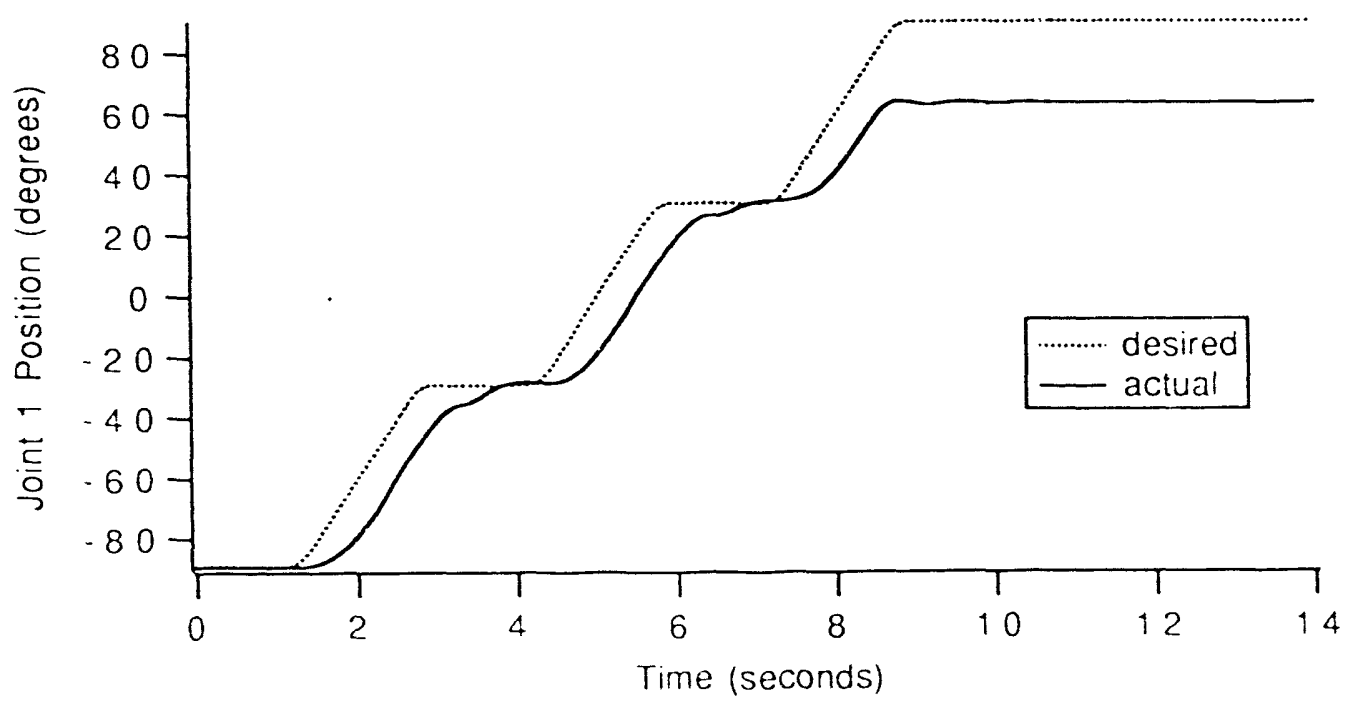

Figure 9. Position of joint 1 during the obstacle avoidance experiment. 

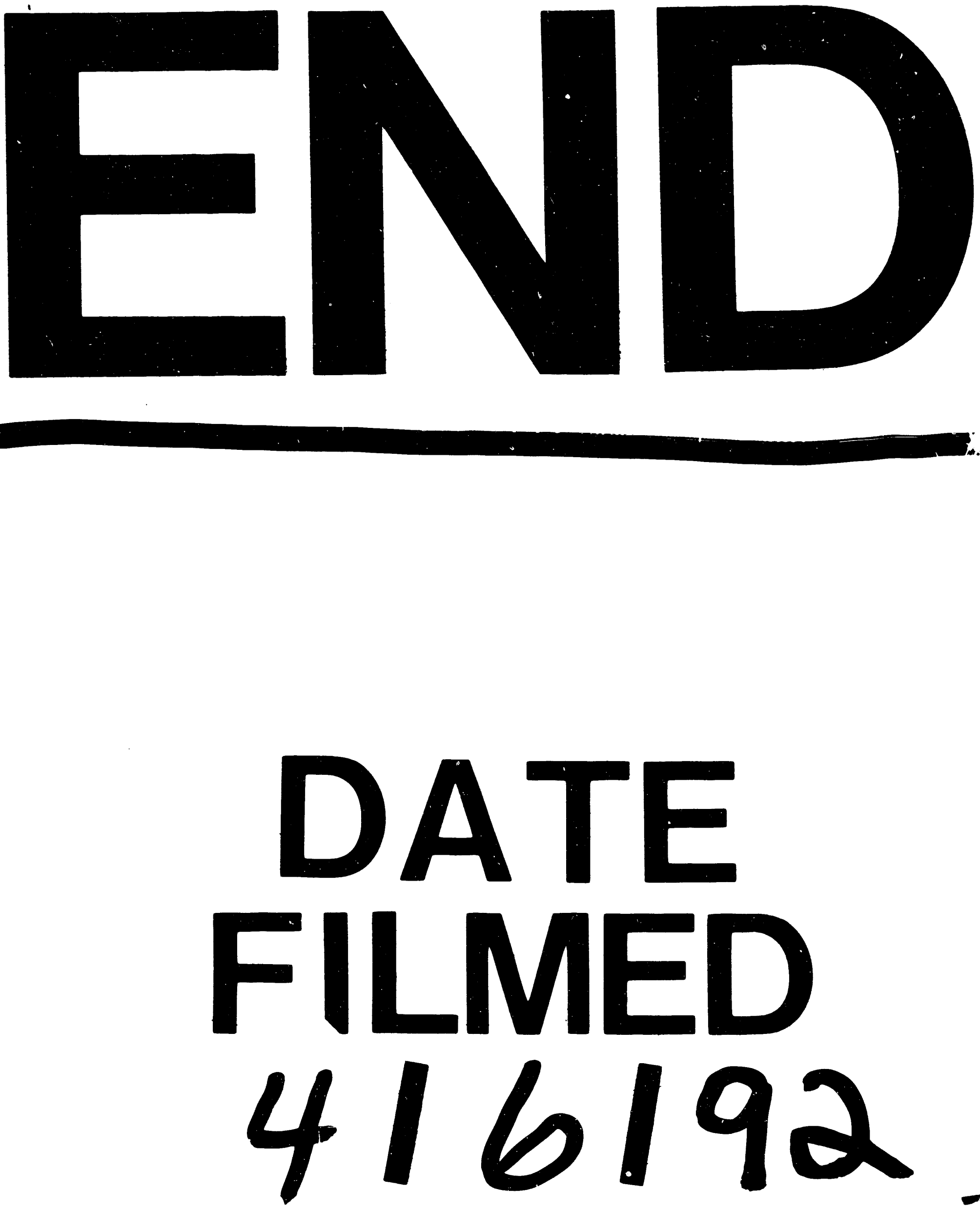

$I$ 
